AIAA-2002-2566

\title{
PHASE SYNCHRONIZATION AND DESYNCHRONIZATION OF STRUCTURAL RESPONSE INDUCED BY TURBULENT AND EXTERNAL SOUND
}

\author{
Lucio Maestrello* \\ NASA Langley Research Center \\ Hampton, VA 23681-2199
}

\begin{abstract}
$\underline{\text { Abstract }}$
Acoustic and turbulent boundary layer flow loadings over a flexible structure are used to study the spatial-temporal dynamics of the response of the structure. The stability of the spatial synchronization and desynchronization by an active external force is investigated with an array of coupled transducers on the structure. In the synchronous state, the structural phase is locked, which leads to the formation of spatial patterns while the amplitude peaks exhibit chaotic behaviors. Large amplitude, spatially symmetric loading is superimposed on broadband, but in the desynchronized state, the spectrum broadens and the phase space is lost. The resulting pattern bears a striking resemblance to phase turbulence. The transition is achieved by using a low power external actuator to trigger broadband behaviors from the knowledge of the external acoustic load inducing synchronization. The changes are made favorably and efficiently to alter the frequency distribution of power, not the total power level. Before synchronization effects are seen, the panel response to the turbulent boundary layer loading is discontinuously spatiotemporally correlated. The stability develops from different competing wavelengths; the spatial scale is significantly shorter than when forced with the superimposed external sound. When the external sound level decreases and the synchronized phases are lost, changes in the character of the spectra can be linked to the occurrence of spatial phase transition. These changes can develop broadband response. Synchronized responses of fuselage structure panels have been observed in subsonic and supersonic aircraft; results from two flights tests are discussed.
\end{abstract}

*Senior Research Scientist, Aerodynamics, Aerothermodynamics, and Acoustics Competency, Associate Fellow AIAA.

Copyright $@ 2002$ by the American Institute of Aeronautics and Astronautics, Inc. The U.S. Government has a royalty-free license to exercise all rights under the copyright claimed herein for Governmental purposes. All other rights are reserved by the copyright owner.

\section{Background}

The interaction of two unrelated nonlinear signals, surface pressure from turbulent boundary layer flow and external high intensity tonal sound, can induce synchronization of the response of a flexible structure. As a result, large amplitude, spatially symmetric loading is superimposed on broadband loading. The history of synchronization goes back to Huygens $1673^{1}$ in his observation of two pendulum clocks. Synchronization which occurs in nonlinear self-sustained oscillators driven by an external periodic force coupled with each other is described by Anishchenko et al. ${ }^{2}$ In the early 1990s, the work of Pecora and Carroll ${ }^{3}$ stimulated interest in the synchronization of chaotic systems. Since then, it has become an active field of research over a range of disciplines including engineering, physics, chemistry, biology, medicine, astronomy. ${ }^{3-8}$ Over the last decade applications have been developed in communication systems where the message is masked by chaos. ${ }^{9}$ Heagy et al. $^{5}$ and Rosenblum et al. ${ }^{6}$ have focused on the instabilities of the synchronous state, which turn out to be very useful for practical applications in nonlinear dynamics. The opposite of phase synchronization is phase desynchronization that is the known physical phenomena in engineering of systems that undergo structural changes due to changes in the degrees of freedom or symmetry and are also used in the study of DNA helix. ${ }^{2}$

The phenomenon of phase synchronization of coupled chaotic systems is presently a topic of great interest. ${ }^{2,5,6}$ Periodic systems are usually called synchronized if either their phase or frequencies are locked. ${ }^{10}$ We demonstrate synchronization from two dissimilar nonlinear chaotic inputs - turbulent boundary layer and exterior tonal sound-when globally coupled to the structure. We show that this behavior is due to synchronization of the phase of the input while the amplitude remains uncorrelated in most cases; ${ }^{6}$ we have found also some cases of frequency synchronization. ${ }^{11}$ 
As a result, the structure becomes highly loaded and coupled to both exterior and interior pressures; the response indicates the presence of harmonics and subharmonics superimposed on broadband. ${ }^{12,13,14}$ In the absence of exterior acoustic forcing, the response of the structure possesses spatiotemporal behavior and shorter spatial scale due to coupling with exterior turbulent boundary layer loading. ${ }^{15,16}$ Synchronization of sound with turbulent and laminar boundary layers also occurs as an interference problem in wind tunnel experiments; this has been a difficult problem.

This paper also describes desynchronized response, which is a result of phase transition designed to redistribute the power into broadband by using a low power actuator to control the response. An alternative method was proposed by Zheng et al. ${ }^{17,18,19}$ The control approach allows us to select and manipulate the outcome of synchronized response from a fixed space point and to estimate the transient time that the trajectories take to converge into the desynchronized state. Non-feedback control is used to perform changes in the system response. This method has been applied in various fields, such as chemical reaction, neuron chaos, electronic systems. ${ }^{20,21,22}$ They have been extended to control high-dimensional systems and applied to other situations such as the synchronization of chaotic systems and the control of spatial systems. Spatial phase synchronization is perceived as increasing aircraft interior noise and structural fatigue, whereas desynchronization has a rather general meaning such as suppressing synchronization when harmful while producing chaos when useful. The time series analysis provides tools to identify dynamical systems from the measured data. The present experiment provides two examples of different structures and external forcing to describe the stability of synchronous oscillation and to demonstrate its applicability.

The first part of this experiment relates to the spatiotemporal dynamics over multidomain regions of space when the panel is forced by turbulent boundary layer. The interactions among these regions lead to spatiotemporal chaos. The second part relates to singledomain response due to synchronization of the turbulent boundary layer with external tonal sound. The third part is on transition from synchronous to asynchronous phase response by forcing the panels with an external force actuator. With knowledge of the initial forcing condition, the response can be manipulated with a very small amount of power to trigger the tonal response into broadband. This paper begins with instrumentation and signal processing in Sec. 2 . Sec. 3 describes the panel response experiment. Analysis and interpretation is discussed in Sec. 4.

\section{Instrumentation and Signal Processing}

The apparatus is an open circuit wind tunnel which has been described at length in previous papers. ${ }^{12}$ The present experiment is conducted on two aluminum aircraft structures of different sizes mounted on the wind tunnel sidewall opposite the anechoic wall, where the acoustic source is located, and is designed to study boundary layer and sound interaction problems. Panel A consists of two flat sections joined by a tear stopper mounted on a rigid baffle (Fig. 1) with measurements along the centerline of the downstream section. Two sections are necessary to allow wave transmission from one to the other. Each section is $0.65 \mathrm{~m}$ long, $0.20 \mathrm{~m}$ wide, $0.001 \mathrm{~m}$ thick, and the tear stopper is $0.0125 \mathrm{~m}$ wide, $0.0128 \mathrm{~m}$ thick. Panel $\mathrm{B}$ is a curved fuselage structure made in six sections with one longitudinal and two lateral tear stoppers equally spaced (Fig. 2). Measurements are made on the lower center section of the panel. Each section is $1.019 \mathrm{~m}$ long, $0.305 \mathrm{~m}$ wide, and $0.109 \mathrm{~cm}$ thick, with a radius of curvature of $2.529 \mathrm{~m}$. The boundaries of the panel with tear stoppers and frame are smooth with a radius of curvature of $0.04 \mathrm{~m}$ to minimize the amplitude of reflected waves at the boundaries. The geometry of the tear stoppers on panel $B$ is a departure from the standard blunt discontinuity used in aircraft and on panel $A$. The Reynolds number per meter $\mathrm{Re} / \mathrm{m}$ is $2.85 \times 10^{5}$, velocity freestream $U_{\mathrm{e}}$ is $46 \mathrm{~m} / \mathrm{s}$, and boundary layer thickness is $0.060 \mathrm{~m}$. The acoustic field is created by four $120-\mathrm{W}$ speakers on the anechoic side with a power level of $138 \mathrm{~dB}$. The forcing frequency of the speakers is set to $f_{1}=475 \mathrm{~Hz}$ for panel $\mathrm{A}$, and $f_{1}=1075$, $f_{2}=1212$, and $f_{3}=1362 \mathrm{~Hz}$ for panel B. Miniature pressure transducers measure the wall pressure fluctuations. The flow velocity is measured by a hot wire anemometer, and the vibration response is measured by miniature accelerometers. The active controller is a feed-forward, open-loop system ${ }^{23}$ freely suspended and mounted at the center of the panel. Accelerometer signals provide the output signal from the panel motion. An accelerometer is also placed at the shaker-panel interface. Data are analyzed to evaluate the response and used to identify nonlinearity. ${ }^{23}$ The study of the dynamical property, Hilbert transform, is used in signal processing. This approach gives the instantaneous phase and amplitude for the signal, which is a complex function of time. ${ }^{7,2}$ 


\section{Response of Panels}

We investigated a scheme for modulating the structural fundamental and formation of harmonic and subharmonic frequencies, as well as for demodulating the response into broadband chaos. Chaos response is a desirable feature in the present application. The first part of the experiment relates the spatiotemporal dynamics over the multidomain region of space when the panel is forced by the turbulent boundary layer. The interaction among these regions leads to spatiotemporal chaos. The second part relates to single-domain response due to synchronization of the turbulent boundary layer and tonal sound loadings, using mono- and threeincommensurate frequency forcing. The third part is on transition from synchronous to asynchronous phase response. As a result, the spectrum broadens by driving the panels with an external force actuator at the initial sound-forcing conditions. The question whether the experiment can be regarded as a representation of inflight aircraft structural response by exploring the nonlinear dynamics is discussed in Sec. 3.6 where flight test data in subsonic and supersonic aircraft are analyzed.

\subsection{Response Due to Turbulent Boundary Layer} Loading

Discrete time measurements of the response are made at different spatial locations using a onedimensional array of accelerometers along a panel centerline. With an appropriate dc bias, one observes the time-dependent propagation and the spatial extent of the nonpropagating waves and the formation of the spatial structure of the surface when forced by the turbulent boundary layer. The continuous change of domain response is an example of spatiotemporal chaos. The pattern is described in terms of amplitude, time, and space along the centerline of the panel and bears a striking resemblance to spatiotemporal chaos turbulence. Data show wide unpredictable ranges of pattern formations with unrepeatable occurrences that coexist and evolve in space-time. Results also show that the spatial scales of the propagating disturbances are less than the boundary layer thickness $\delta(y)$ and much less than the length of the panel. Space-time response indicates reduction in amplitude with distance downstream due to the suppression of the growth of the higher frequency components. The spatial and temporal responses of the panel wave are not separable, the spatial evolution can affect the temporal behavior and vice versa.
The acceleration responses $g(x, t)$ and the phase $d g(x, t) / d t$ versus $g(x, t)$ over the time interval $\Delta t=0.04 \mathrm{sec}$ along the panel centerline of panel $\mathrm{A}$, at four equally spaced locations $x=0.00624,0.1248$, $0.1822,0.2196 \mathrm{~m}$ are shown in Figs. $3 \mathrm{a}$ and $3 \mathrm{~b}$. The vibrating motion is induced by the fluid-elastic coupling of the turbulent shear layer. The data indicate distinct propagating wave patterns as well as nonpropagating ones emitting disturbance very slowly with time. The nonpropagating pattern coexists over a larger portion of time before switching into propagating patterns again by superimposition. The propagation disturbances have velocity less than the boundary layer freestream velocity. The result contradicts time-averaged space-time correlation measurements which show continuous wellbehaved convection patterns.

\subsection{Spatial Phase Synchronization Induced by Turbulent Boundary Layer and Sound Forcing}

Synchronized response of a typical aircraft panel structure results from the interaction between an external tonal sound and a turbulent boundary layer forcing a structure. The degree to which the panel oscillations adjust to a synchronous response depend on the degree to which the forcing adjusts to the panel motion.

Phase synchronization is associated with spatial ordering of the phase induced by turbulent boundary layer $\phi_{1}(t)$ with external sound phase $\phi_{2}(t)$ on the structure. As a result, the phase difference is

$$
\phi_{n, m}(t)=n \phi_{1}(t)-m \phi_{2}(t)=\text { Constant }
$$

where $\phi_{1}$ and $\phi_{2}$ are phases, $n$ and $m$ are integers, and $\phi_{m, n}$ is the generalized phase difference. This condition is valid for quasi-periodic oscillations only with two incommensurate frequencies. The second type corresponds to phase locking described by Rosenblum et al. ${ }^{6}$ in coupled chaotic system as

$$
\left|n \phi_{1}(t)-m \phi_{2}(t)\right|<\text { Constant }
$$

the amplitudes of the two systems may be completely uncorrelated, that is, linearly independent. Note that for the determination of synchronous states it is irrelevant whether the amplitudes of both inputs are different. Alternatively one can use the concept of frequency synchronization if the weaker condition 11

$$
\Gamma_{n, m}(t)=<n \phi_{1}(t)-m \phi_{2}(t)>=0
$$

is satisfied; $<>$ denotes time average. 
Experimentally, the phase due to the interaction of the flow and external sound slips. Reconstructing the phase synchronization from experimental data has a limit, since the total phase is made up of coherent, average, and random fluctuating parts as indicated by Anishchenko et al. ${ }^{2}$ and Pikovsky et al. ${ }^{7}$ The experimental phase synchronization is characterized by a sharp peak response, whereas the asynchronous response spreads over a broadband at nearly equal power. Synchronization is a function of the coupling strength because of particular instability driven by long acoustic wavelength on the turbulent boundary layer forcing the structure.

The flow speed and tonal sound are kept low in the beginning. As time progresses, the flow and acoustic loading are gradually increased until the panel reaches a transition into a synchronized response. During the transition, the response remains synchronized for a certain period of time, becomes unsynchronized, and then becomes synchronized again. After further increases in tonal level, the response becomes nearly continuous to permanently synchronized. At fixed forcing frequencies as the flow velocity increases to the constant value, the formation of harmonics and subharmonics of the driving frequency and the phase locking on the panel superimposed on the broadband turbulent boundary layer loading are observed. The synchronized spatial pattern is attributed to high acoustic loading and to the spatial scale of the tonal sound relationship to the spatial scale of the convected turbulent boundary layer flow.

\subsection{Monofrequency Forcing Turbulent Boundary} Layer-Panel A

Measurements for panel $\mathrm{A}$ are made in real time at three equally spaced locations along the centerline. The high amplitude tonal sound on turbulent boundary layer at frequency $f_{1}=475 \mathrm{~Hz}$ leads to a spatial phase synchronization response. The synchronized phase is characterized by many closely spaced peaks resulting from the modulation in the phase and time domains. The combination of the acoustic and turbulent pressure fluctuations gives rise to the time series of the acceleration response $g(x, t)$ representing the motion of the surface. The corresponding computed power spectral density $G(x, T)$ and the total phase $d g(x, t) / d t$ versus $g(x, t)$ are shown in Figs. $4 \mathrm{a}$ and $4 \mathrm{~b}$. The figures describe the main feature of the dynamics. The response seems to consist of harmonic waves coupled with random fluctuating amplitude and bandwidth. Data from selected locations show that their motions are temporally chaotic but spatially periodic.
An increase in synchronization response, that is, a measurement of phase coherence, is regarded as a state of increased acoustic loading that will induce coupling with the turbulent flow; thus, the response of the structure is affected. The phase space measured over finite time is also nearly periodic with superimposed irregular modulations, concentrated over bandwidths, whereas outside the phase difference, changes are nearly continuous and averaged approximately over $2 \pi$. The phase spreading is due to the lack of phase synchronization leading toward phase turbulence. The synchronized spectrum is broadband with superimposed sharp periodic peaks indicating dominant frequencies. The windows of chaos are apparent between synchronized peaks. The broadening of the peak bandwidth can be thought of as an amplitude modulation of the spectrum of an otherwise periodic response. As a result the power spectra plots contain superimposed peaks and bandwidth modulation; this leads to the conclusion that the panel surface maintains periodic response with oscillating peak amplitude and bandwidth from location to location. The intensity distribution decreases with harmonic order irregularity, perhaps because of phase-amplitude-frequency mismatch between harmonic bands due to depletion of nonlinearity. Spatial symmetry imposes stringent conditions on the dynamics response of the structure; when symmetry is slightly broken because of an increase in external sound level, one observes complicated quasi-periodic behavior. The spectrum of the response leads to the conclusion that the panel surface maintains periodic response with oscillating peak amplitude and bandwidth from location to location.

The results exhibit spatial phase synchronization, which has not been studied theoretically. The plots (Fig. 4) are obtained from the short time signal processes of the time series data; the phase and spectra peaks, due to temporal superposition of events, contain coherent, average, and random fluctuation parts as interpreted by Pikovsky et al. ${ }^{7}$ The chaotic peak and bandwidth modulations create a complex phase. Different ways have been used to define the phase in signal analysis. The problem is discussed in Sec. 4.

\subsection{Multiple Frequencies Forcing Turbulent Boundary Layer-Panel B}

Measurements of the panel response are made simultaneously at four equally spaced points along the centerline of the bottom center panel (Fig. 2). Figs. 5a and $5 \mathrm{~b}$ show the acceleration response $g(x, t)$ of the time series over the time interval $t$ and the computed power 
spectral density $G(f, T)$. The tonal sound is superimposed on the turbulent boundary layer broadband response generated of three incommensurate frequencies $f_{1}=1075, f_{2}=1212$, and $f_{3}=1362 \mathrm{~Hz}$. The time series is made up by wave packet response. The modulations in the series have been previously observed ${ }^{23,24}$ to be the result of frequency locking due to the intermix periodic and nonperiodic phases and frequencies. ${ }^{23} \mathrm{~A}$ particular feature of the power spectra is the appearance of incommensurate frequencies mixed with harmonics and subharmonics. As a result, differences in response between locations are mainly on the association of the harmonics and subharmonics transient due to unstableunstable fluid and acoustic forcing. ${ }^{22}$ The two inputs, the turbulent boundary layer and sound, are coupled; synchronization in their dynamical variables has occurred because of coupling strength induced by their unstable pair of inputs. Observations from different data runs have indicated the occurrence of a nonuniqueness spatial-temporal response between runs. An example, the plots in Fig. 5, indicates a temporal shift between the top two locations (interpreted convective effects), whereas at the bottom two locations it is weakly correlated and frozen in space.

\subsection{Phase Desynchronization Induced by External} Forcing

The desynchronization of a synchronous response is a changed power distribution from peak level to broadband level using active external forcing. A small shaker attached to the panel provides the external force.

Control of a dynamic system, via phase transition, has to be associated with improved performance to be of value in aircraft applications. Control of the peak spectral density of a synchronized response is a loss of synchronization, which gives rise to multiscaling and phase turbulence. The changes of the phase from partially synchronized to synchronized are shown in Figs. $6 \mathrm{a}$ and $6 \mathrm{~b}$ for panel $\mathrm{A}$ at location $x=0.02331 \mathrm{~m}$. The spectra also reduced to broadband level, via partially synchronized into desynchronized state, are shown in Figs. $6 \mathrm{c}$ and $6 \mathrm{~d}$ compared with the synchronized response. The initial tonal forcing triggers changes in the response redistributing the energy of the fundamental and harmonics into broadband. In this experiment, the property of the initial forcing relates to frequency, amplitude, and phase of the fundamental tones at each fixed-point measurement on the panel. ${ }^{12,27,22,28}$ The broadband spectra of the panel response has lower amplitude than the synchronized spectra; the change in response is obtained without significant change in total response power. The spectra peak level reduces about $15-\mathrm{d} \mathrm{B}$ power on the average. The integrated spectra level indicates that a small amount of energy is lost in the process because of the broadband redistribution. The resulting response is made up of a large number of unstable orbits, similar to the response when the structure is forced by turbulent boundary layer alone. The initial tonal forcing was found to be an effective trigger of changes in the redistributing energy favorably and efficiently. ${ }^{27}$ The stability of the fundamentals and harmonics, as well as incommensurable frequencies, not shown, play a large role on the active control dynamics. The enhanced stability seems to be the breakup of large spatial domain of the synchronized response into smaller space domain. The synchronized control responses, originated from different runs, cannot be duplicated experimentally. Spatial domain is large enough that the boundary spatial effects can be neglected when the response is synchronized. The breakup of the synchronized domain leads to a progressive collapse of the spatial domain. The spatially synchronized perturbations have larger amplitude than the asynchronous spatiotemporal perturbations.

\subsection{Flight Test Measurements}

Synchronized responses from measurements on selected airplane fuselage structure panels in subsonic and supersonic flights are discussed.

3.6.1. Subsonic flight. The data analyzed on a Boeing MD-90 (derivative of DC-9) fuselage panel were recorded at Mach number 0.80 and an altitude of $10,000 \mathrm{~m}$, by Mathur et al. ${ }^{29}$ The response of the accelerometer 41 , located $8 \mathrm{~m}$ downstream on the right side of the fuselage, was analyzed. The analysis is from the time series using short-time signal processing. The series indicates the occurrence of sharp periodic peaks intermixed with nonperiodic ones. The peaks and bandwidth oscillate because of the nonstationarity of the input. The time series, the computed spectrum, and phase responses are shown in Figs. $7 \mathrm{a}, 7 \mathrm{~b}$, and $7 \mathrm{c}$. Periodic peaks at approximately a $263-\mathrm{Hz}$ interval dominate the response; the amplitude levels exceed the broadband level by $10-\mathrm{dB}$ power. Note that few peaks dominate the response, due to turbulent boundary layer loading alone, because the measurement location is remote from engine noise and wing interference. The phase indicates convection; the disturbances propagate along the direction of flow. The panel response is not synchronized, as in the previous experiments in 
Secs. 3.3, 3.4, and 3.5. The peak frequency loadings are associated with both incommensurate and commensurate frequencies; the dominant peaks are periodic. Because the initial forcing was established to be the origin of the response state, control can be applied to the original orbits, which can yield an improved performance or steady state. A single low power activator on the panel may control the peak periodicity loading that propagates over the entire surface 27,30 . Since 1971, from measurements on a Boeing $727^{31}$ at Mach number 0.85, only a few modes were known to dominate the response and interior noise level toward the front part of the fuselage; this is consistent with present measurements.

3.6.2. Supersonic flight. The data analyzed on a panel structure of a Tu-144LL supersonic transport was recorded at Mach number 1.95 and an altitude of $17,000 \mathrm{~m}$ and reported by Rizzi et al. ${ }^{32}$ The data are from an accelerometer above the wing and window on the right side of the fuselage, location 10.15. The data were analyzed from the time series; the computed power spectrum and phase responses are shown in Figs. 8a, $8 \mathrm{~b}$, and $8 \mathrm{c}$. The three plots indicate nonperiodic, nonstationary behaviors, well-defined spectra peaks, and the phase indicative of convection waves on the panel surface propagating along the direction of flow. ${ }^{33}$ With regard to the time history plots, successive time records indicate portions which are solely characterized by turbulent boundary layer loading (Fig. 8d) and other portions can be characterized by superposition of moving shock on turbulent boundary layer (Fig. 8e). The superposition of shocks with turbulent boundary layer can induce a synchronized response.,6,7 The results are not surprising, because the pressure above the wing is known to have nonuniform distribution, due to compression-expansion waves temporally superimposed on turbulent boundary layer.

\section{Analysis and Interpretation of Experimental Data}

A prediction of the evolution of the driven system based on data from the response is possible because any time series measured at the response may be viewed as a time series from the combined systems, drive and response, and may, thus, be used to reconstruct and predict the dynamics of drive and response. ${ }^{22}$ For small coupling, the response of the panel structure undergoes a transition from synchronized state to a chaotic state or spatially disordered phase. These phases indicate that the synchronous state is self-sustained.

\subsection{Panel Response Spectrum}

Consider the motion of $n$ points on a nonlinear flexible panel at $x=x_{1}, x_{2}, \ldots, \mathrm{x}_{n}$, subject to the loading by the pressure fluctuation $p_{T}\left(x_{i}, t\right)$ in the turbulent boundary layer and an external periodic acoustic excitation $p_{A}\left(x_{i}, t\right)$ at $x=x_{i}$ (Fig. 9). As a simple model, assume that the motion of the point $\mathrm{x}_{i}$ is governed by a nonlinear differential equation,

$$
\begin{aligned}
& \ddot{u}_{i}(t)+\mu \dot{u}_{i}(t)+r_{i}\left(w_{i}\right)= \\
& p_{i}(t)+q_{i}(t) \quad(i=1,2, \ldots, n)
\end{aligned}
$$

where $u_{i}$ is panel displacement; $r_{i}$ is nonlinear elastic restoring force, which, for large separation of points, has no interaction with other points; $p_{i}(t)=p_{T}\left(x_{i}, t\right)$ and $q_{i}(t)=p_{A}\left(x_{i}, t\right)$ where $i=1,2, \ldots, n$, and $\mu$ is the damping coefficient. For a fixed point $x_{i}$, by dropping the index $i$, equation (1) can be rewritten as

$$
\ddot{u}(t)+\mu \dot{u}+r(u)=p(t)+q(t)
$$

Here, the turbulent pressure $p(t)$ is a random function of $t$ and the acoustic pressure $q(t)$ is given by

$$
q(t)=A \cos \theta(t)
$$

where the phase $\theta(t)=\omega t+\theta_{0}$, the amplitude $A$ and the frequencies $\omega$ are positive constants, and $\theta_{0}$ is the initial phase. As commonly assumed, for a nonlinear beam, the nonlinear restoring force $r(u)$ is close to a cubic function. For a nonlinear beam, Homes et al. ${ }^{28,34}$ gives

$$
r(u)=k^{2} u-\alpha u^{3}+\mathrm{O}\left(u^{4}\right) \quad(k>0, \alpha>0)
$$

where the term of $\mathrm{O}(u)^{4}$ and higher will be neglected.

In view of Equations (3) and (4), Equation (2) yields

$$
\ddot{u}+\mu \dot{u}+k^{2} u-\alpha u^{3}=A \cos \left(\omega t+\theta_{0}\right)+p(t)
$$

Suppose there is no flow excitation, $p(t)=0$. It is wellknown that the simple harmonic acoustic forcing can generate harmonic response as

$$
\begin{aligned}
u(t)= & u_{A}(t)=A_{1} \cos \left(\omega t+\theta_{1}\right)+A_{2} \cos \left(2 \omega t+\theta_{2}\right) \\
& +\ldots+A_{n} \cos \left(n \omega t+\theta_{n}\right)+\ldots
\end{aligned}
$$

Without the acoustic excitation $(A=0)$, the solution $u_{T}(t)$ of Equation (5) exhibits a broadband random signal. The combination of the acoustic and turbulent pressure excitations gives rise to the acceleration spectrum $G(f, T)$ of the response $u(t)$ as shown in Fig. 4a. The response consists of harmonic response 
embedded in the randomly fluctuating signal (without control).

\subsection{Phase Synchronization}

The experimental data (Fig. 4a) show that for $n=3$, the acceleration spectra are similar for three cases at $x=x_{1}, x_{2}, x_{3}$ as far as the phase $\psi(t)$ of the periodic component is concerned. The results exhibit a spatial phase synchronization, which has not yet been studied theoretically. The notion of phase synchronization implies interaction between phases of two self-sustained oscillators where the amplitude can be uncorrelated, a concept introduced by Pikovsky et al. ${ }^{35}$ At a fixed point, the phase synchronization in the presence of noise, such as Equation (5), has been investigated by several authors (Anishchenko et al. ${ }^{2}$ ). For simplicity, set $\theta_{0}=0$, and write

$$
u(t)=b(t) \cos \psi(t)
$$

where $b$ and $\psi$ are the amplitude and phase for the response signal. It is possible to obtain a first-order, coupled equation for $b$ and $\psi$ (Anishchenko et al. ${ }^{2}$ ). However, as a simple approximation, one can neglect the slow amplitude variations. See Pikovsky et al. ${ }^{7}$ to get the stochastic equation for the phase difference $\phi(t)=\psi(t)-\theta(\mathrm{t})$ as follows:

$$
\phi(t)=(\Omega-\omega)-G(\phi)+\xi(t)
$$

where $\Omega$ is the frequency of free oscillation for the nonlinear Equation (5), $G$ is a $2 \pi$ periodic function, and $\xi(t)$ is a random process function. Due to the random perturbation $\xi(t)$, the phase difference $\phi(t)$ is incoherent and shows random fluctuation. Therefore the total phase $\psi(t)=\theta(t)+\phi(t)$ consists of a coherent part, the average phase $\langle\psi(t)\rangle$ plus a random fluctuation as shown in Fig. 4b.

The temporal oscillations over spatially selected points on the panel A were evaluated. To investigate the problem of phase synchronization one can apply the method introduced by Rosenblum et al. ${ }^{6}$ and Pikovsky et al. ${ }^{7}$ Using this approach, the temporal difference $\Delta \phi(t)=\phi_{1}(t)-\phi_{2}(t)$ between the instantaneous phases $\phi_{1}(t)$ and $\phi_{2}(\mathrm{t})$ of the coupled response can be followed. The method consists of extracting the phase of the scalar $s(t)$ and amplitude $A(t)$ via the construction of the analytic signal which is a complex function of time defined as

$$
s(t)+j \bar{s}(t) \equiv A(t) e^{j \phi(t)}
$$

where

$$
\bar{s}(t)+\frac{1}{\pi} \mathrm{PV} \int_{-\infty}^{\infty} \frac{s(\tau)}{t-\tau} d t
$$

denotes the Hilbert transform of $s(t), j$ is the imaginary unit, and PV means that the integral is taken in the sense of the Cauchy principal value. The instantaneous amplitude $A(t)$ and the instantaneous phase $\phi(t)$ of the signal $s(t)$ between two inputs are

$$
\phi_{n, m}(t)=\left|n \phi_{1}(\mathrm{t})-m \phi_{2}(t)-\delta\right|<\mu
$$

where $\mu$ is a small parameter $(\mu<2 \pi)$ and $\delta$ is average phase shift. Generally the relative phase difference remains bound to small interval $\mu$ and mean value $\delta$ during the synchronous state, Equation (9) corresponding to phase locking. ${ }^{35}$ In our system one can expect the condition of phase synchronization to be satisfied over a finite time. The phase difference $\left|\phi_{1}-\phi_{2}\right|$ for $\delta=0.03$ of the unsteady acceleration response from the time series of Fig. 4a, $x=0.1552 \mathrm{~m}$, is shown in Fig. 10. The phase difference of the lowest modes oscillates over a small angular difference; however, the tendency to destabilize can be seen as $t$ increases. The synchronous state is again recovered as time increases.

\section{Conclusions}

We have described a wind tunnel experiment of spatial phase synchronization of flexible structures where the amplitude-bandwidth oscillations are chaotic and the phase synchronized. The response emerges from two nonidentical chaotic inputs, acoustic, and turbulent boundary layer loading. The response is desynchronized into broadband chaos by active forcing. A single, low power external actuator is sufficient to overcome the spatial response of synchronization; this results in a redistribution of tonal components into broadband at nearly equal power, a spatiotemporal chaotic behavior. Synchronization without external coupling is not possible; also maintaining broadband chaos from synchronization without external forcing is not possible. The conclusions are summarized as follows:

1. Reconstructing phase synchronization from experimental data that are used to identify the dynamics has limits, since the total phase is made up of coherent, average, and random fluctuating parts. 
2. Phase synchronization due to small changes in system input cause large variations in response.

3. The loading and response mechanisms described are verifiable and pertain to that encountered on contemporary aircraft.

4. The power required to induce desynchronization in the present system is estimated to be 10 percent of the total but the power required to maintain it is less.

5. The controller is simple and easy to realize.

6. The results exhibit spatial phase synchronization which has not yet been studied theoretically.

7. Control of the synchronized response, from two chaotic inputs, can perhaps be extended to trigger further changes toward an initial periodic state.

\section{$\underline{\text { References }}$}

1. Huygens, C., "Horologium Oscillatorium," Apud F. Muguet, Paris, 1673.

2. Anishchenko, V. S., Vadivasova, T. E., Postnov, D. E., and Safonova, M. A., "Synchronization of Chaos," International Journal of Bifurcation and Chaos, Vol. 2, No. 3, 1992, pp. 633-644.

3. Pecora, Louis M., and Carroll, Thomas L., "Synchronization in Chaotic Systems," Physical Review Letters, Vol. 64, No. 8, 1990, pp. 821-824.

4. Rulkov, Nikolai F., Sushchik, Mikhail M., Tsimring, Lev S., and Abarbanel, Henry D. I., "Generalized Synchronization of Chaos in Directionally Coupled Chaotic Systems," Physical Review E, Vol. 51, No. 2, 1995, pp. 980-994.

5. Heagy, J. F., Carroll, T. L., and Pecora, L. M., "Synchronous Chaos in Coupled Oscillator Systems," Physical Review E, Vol. 50, No. 3, 1994, pp. 1874-1885.

6. Rosenblum, Michael G., Pikovsky, Arkady S., and Kurths, Jürgen, "Phase Synchronization of Chaotic Oscillator," Physical Review Letters, Vol. 76, No. 11, 1996, pp. 1804-1807.

7. Pikovsky, Arkady S., Rosenblum, Michael G., Osipov, Grigory V., and Kurths, Jürgen, "Phase Synchronization of Chaotic Oscillators by External Driving," Physica D, Vol. 104, No. 3-4, 1997, pp. 219-238.
8. Hu, Gang, Zhang, Ying, Cerdeira, Hilda A., and Chen, Shigang, "From Low-Dimensional Synchronous Chaos to High-Dimensional Desynchronous Spatiotemporal Chaos in Coupled System," Physical Review Letters, Vol. 85, No. 16, 2000, pp. 3377-3380.

9. Terry, John R., and VanWiggeren, Gregory D., "Chaotic Communication Using Generalized Synchronization," Chaos, Solitons \& Fractals, Vol. 12, No. 1, 2001, pp. 145-152.

10. Tass, P., Rosenblum, M. G., Weule, J., Kurths, J., Pikovsky, A., Volkmann, J., Schnitzler, A., and Freund, H.-J., "Detection of $n: m$ Phase Locking From Noisy Data: Application to Magnetoencephalography," Physical Review Letters, Vol. 81, No. 12, 1998, pp. 3291-3294.

11. Holstein-Rathlou, Niels-Hanrik, Yip, Kay-Pong, Sosnovtseva, Olga V., and Mosekilde, Erik, "Synchronization Phenomena in Nephron-Nephron Interaction," Chaos, Vol. 11, No. 2, 2001, pp. $417-426$.

12. Maestrello, L., "Active Control of Panel Vibration Induced by Accelerated Turbulent Boundary Layer and Sound," AIAA Journal, Vol. 35, 1997, pp. 796-801.

13. Dowell, E. H., "Aeroelasticity of Plate and Shells," Noordhoff International Publishing, Leyden, 1975.

14. Reynolds, R. R., and Dowell, E. H., "Nonlinear Aeroelastic Response of Panels," AIAA Paper 93-1599-CP, 1993.

15. Chow, P. L., and Maestrello, L., "Vibration Control of a Non-Linear Elastic Panel," International Journal of Non-Linear Mechanics, Vol. 36, No. 4, 2001, pp. 709-718.

16. Ting, L., "Boundary Layer Over a Flat Plate in Presence of Shear Flow," Physics of Fluids, Vol. 3, 1960, pp. 78-81.

17. Zheng, Zhigang, $\mathrm{Hu}, \mathrm{Bambi}$, and $\mathrm{Hu}$, Gang, "Collective Phase Slips and Phase Synchronizations in Coupled Oscillator Systems," Physical Review E, Vol. 62, No. 1, 2000, pp. 402-408.

18. Zheng, Zhigang, Hu, Gang, and Hu, Bambi, "Phase Slips and Phase Synchronization of Coupled Oscillators," Physical Review Letters, Vol. 81, No. 24, 1998, pp. 5318-5321.

19. Hu, Bambi, and Zheng, Zhigang, "Phase Synchronizations: Transitions From High- to Low- 
Dimensional Tori Through Chaos," International Journal of Bifurcation and Chaos, Vol. 10, No. 10, 2000, pp. 2399-2414.

20. Jackson, E. A., "On the Control of Complex Dynamical System," Physica D, Vol. 50, 1991, pp. 341-366.

21. Lima, Ricardo, and Pettini, Marco, "Suppression of Chaos by Resonant Parametric Perturbations," Physical Review A, Vol. 41, No. 2, Jan. 1990, pp. $726-733$.

22. Coombers, S., and Lord, G. J., "Desynchronization of Pulse-Coupled Integrated-and-Fire Neurons," Physical Review E, Vol. 55, No. 3, 1997, pp. 55-57.

23. Maestrello, L., "Controlling Vibrational Chaos of a Curved Structure," AIAA Joumal, Vol. 39, No. 4, 2001, pp. 581-589.

24. Gao, J. Y., Narducci, L. S., Schulman, M., and Yuan, J. M., "Route to Chaos in a Bistable System With Delay," Physical Review, Vol. A28, No. 5, 1983, p. 2910.

25. Maza, D., Vallone, A., Mancini, H., and Boccaletti, S., "Experimental Phase Synchronization of a Chaotic Convective Flow," Physical Review Letters, Vol. 85, No. 26, 2000, pp. 5567-5570.

26. Yanchuk, Sergiy, Maistrenko, Yuri, Lading, Brian, and Mosekilde, Erik, "Effects of a Parameter Mismatch on the Synchronization of Two Coupled Chaotic Oscillators," International Journal of Bifurcation and Chaos, Vol. 10, No. 11, 2000, pp. 2629-2648.
27. Maestrello, L., "The Influence of Initial Forcing on Non-Linear Control," Journal of Sound and Vibration, Vol. 239, No. 4, 2001, pp. 873-883.

28. Holmes, P. J., and Whitley, D. C., "On the Attracting Set for Duffing's Equation, I: Analytical Methods for Small Force and Damping," Partial Differential Equations and Dynamical Systems, Edited by W. E. Fitzgibbon III, Pitman, London, 1984, pp. 211-240.

29. Mathur, G. P., Tran, B. N., and Simpson, M. A., "MD-90 Cabin Noise Diagnostics Flight Test," Rept. CRAD-9402-TR-4885, Boeing Co. (Contract NAS1-20268), Oct. 1998.

30. Maestrello, L., "Active Control by Conservation of Energy Concept," AIAA Paper 2000-2045, June 2000.

31. Bhat, W. V., and Wilby, J. F., "Interior Noise Radiation by an Aircraft Fuselage Subjected to Turbulent Boundary Layer Excitation and Evaluation of Noise Reduction Treatments," Journal of Sound and Vibration, Vol. 18, No. 4, 1971, pp. 449-464.

32. Rizzi, S. A., Rackl, R. G., and Andrianov, E. V., "Flight Test Measurements From the Tu-144LL Structure/Cabin Noise Experiment," NASA/TM2000-209858, 2000.

33. Frendi, A., "Coupling Between a Supersonic Turbulent Boundary Layer and Flexible Structure," AIAA Journal, Vol. 35, No. 1, 1997, pp. 58-56.

34. Holmes, P. J., and Rand, D. A., "Phase Portraits and Bifurcations of the Nonlinear Oscillator." International Journal of Non-Linear Mechanics, Vol. 15, 1980, pp. 449-458.

35. Pikovsky, Arkady, Rosenblum, Michael, and Kurths, Jürgen, "Phase Synchronization in Regular and Chaotic Systems," International Journal of Bifurcation and Chaos, Vol. 10, No. 10, 2000, pp. 2291-2305. 


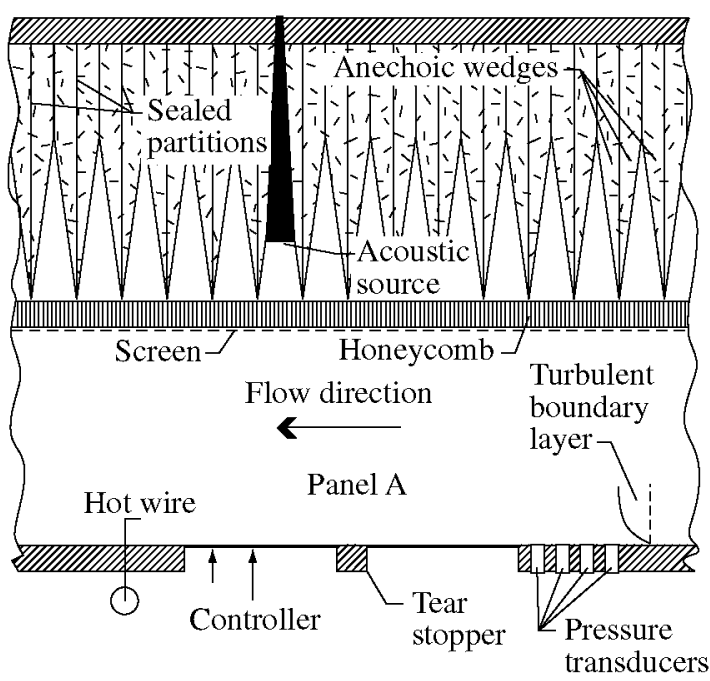

Figure 1. Wind tunnel setup with flat panel A.

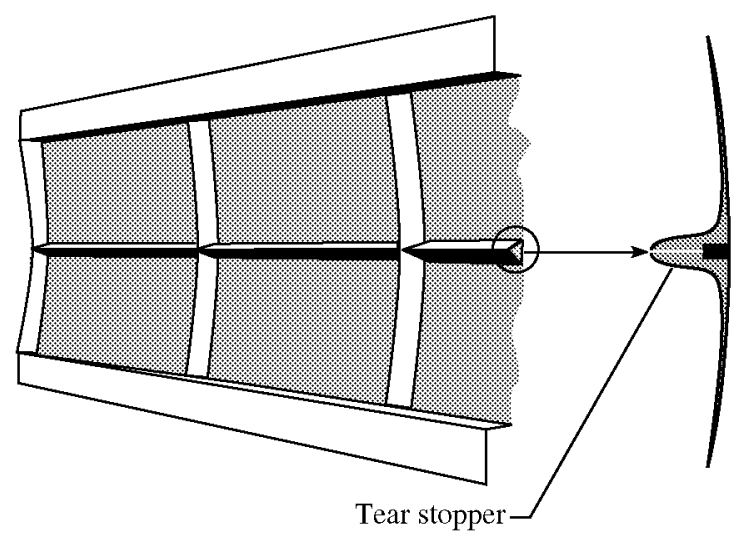

Figure 2. Curved panel B. 


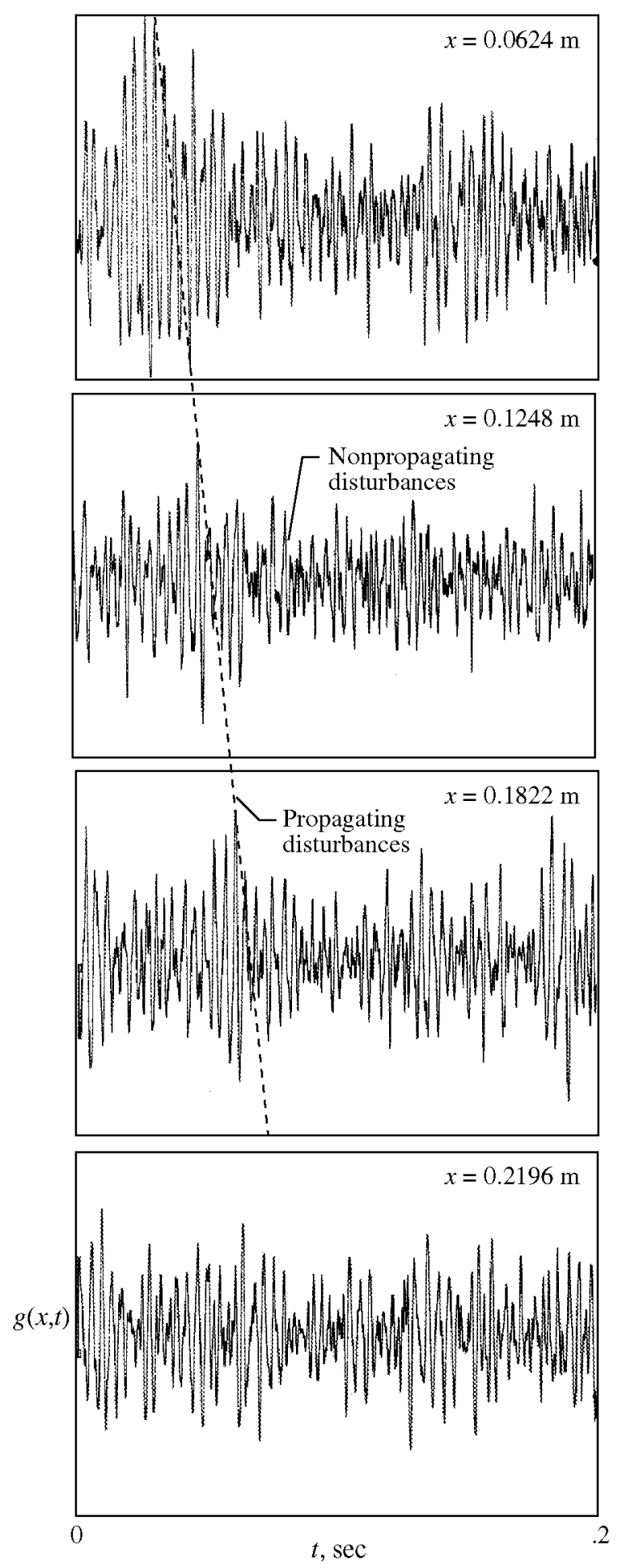

(a) Time series.
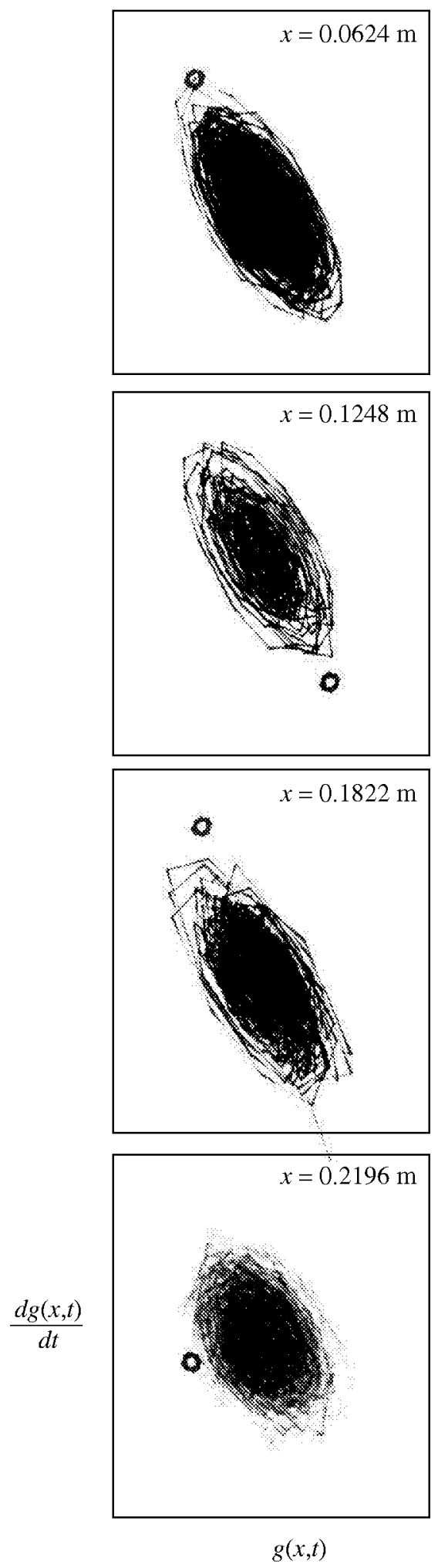

(b) Phase.

Figure 3. Response due to turbulent boundary layer loading at four locations on panel A. 

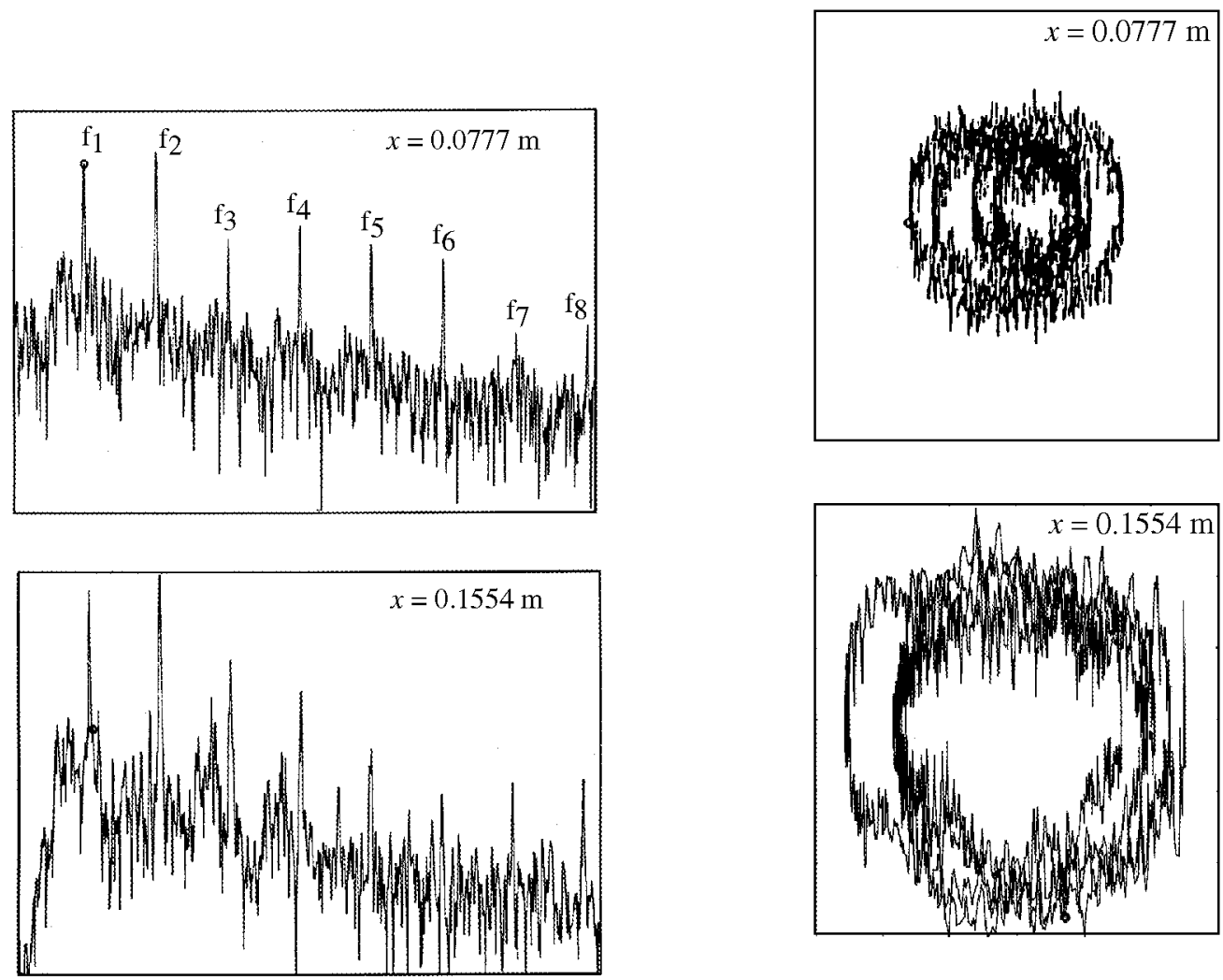

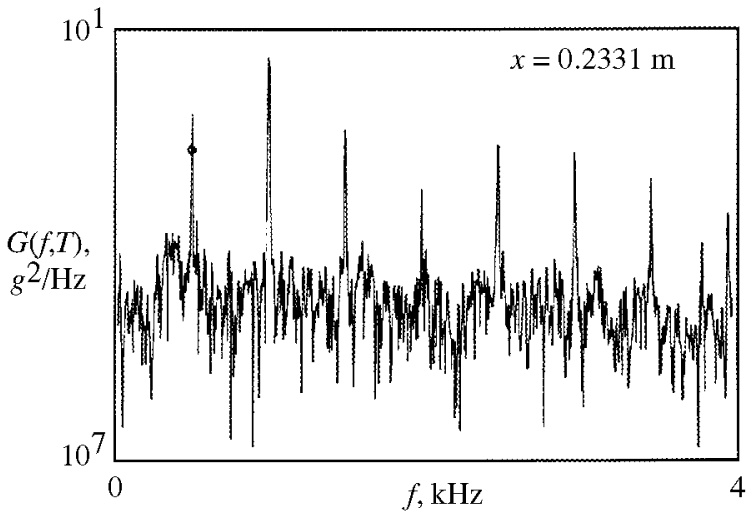

(a) Power spectral density.

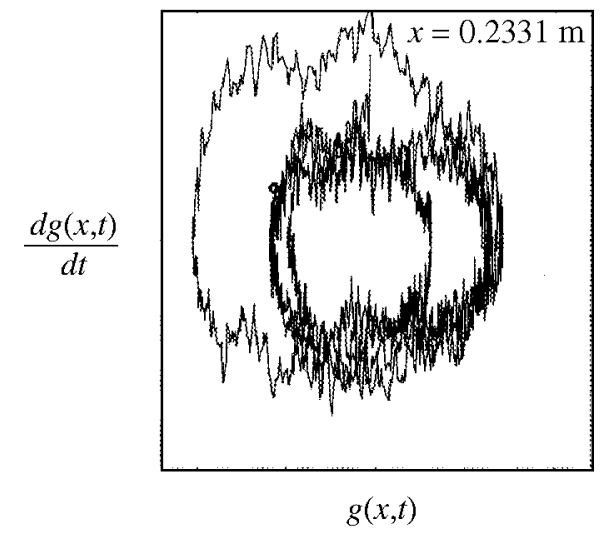

(b) Phase.

Figure 4. Response due to turbulent boundary layer and tonal sound loadings at three locations on panel A. 

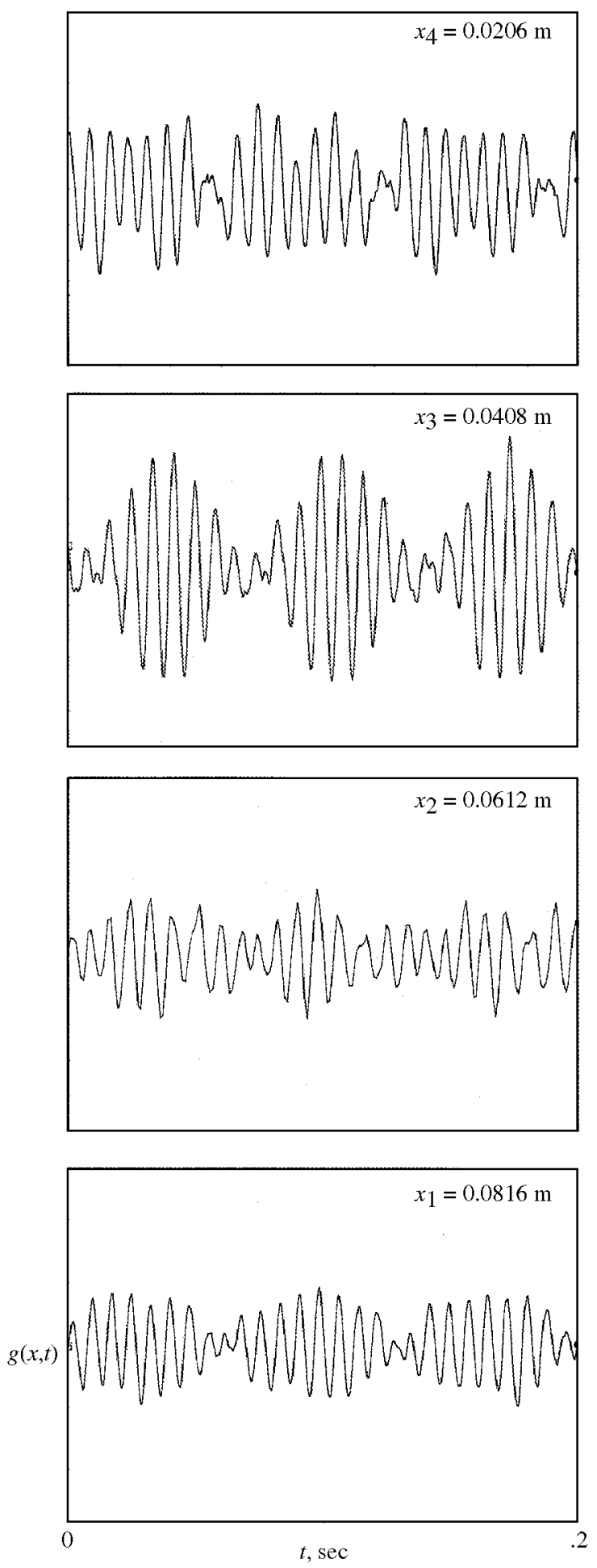

(a) Time series.
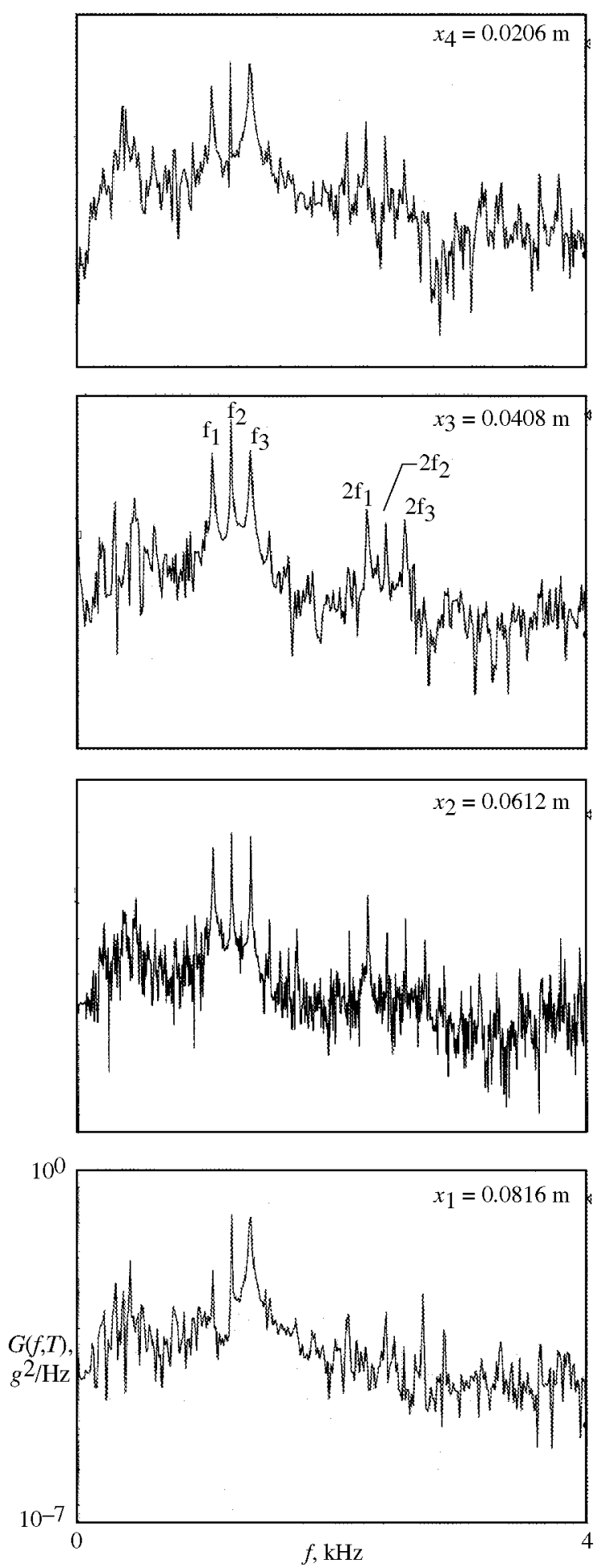

(b) Power spectral density.

Figure 5. Response due to turbulent boundary layer and tonal sound loadings at four locations on panel B. 


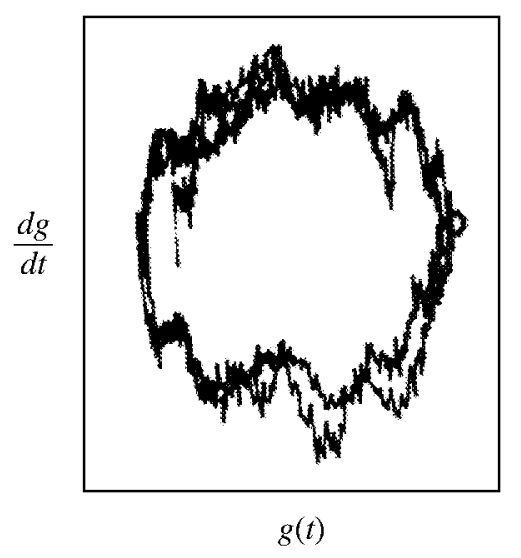

(a) Partially synchronized phase.

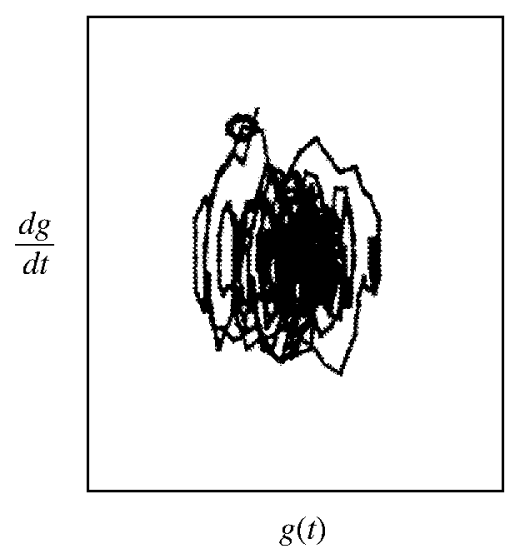

(b) Desynchronized phase.

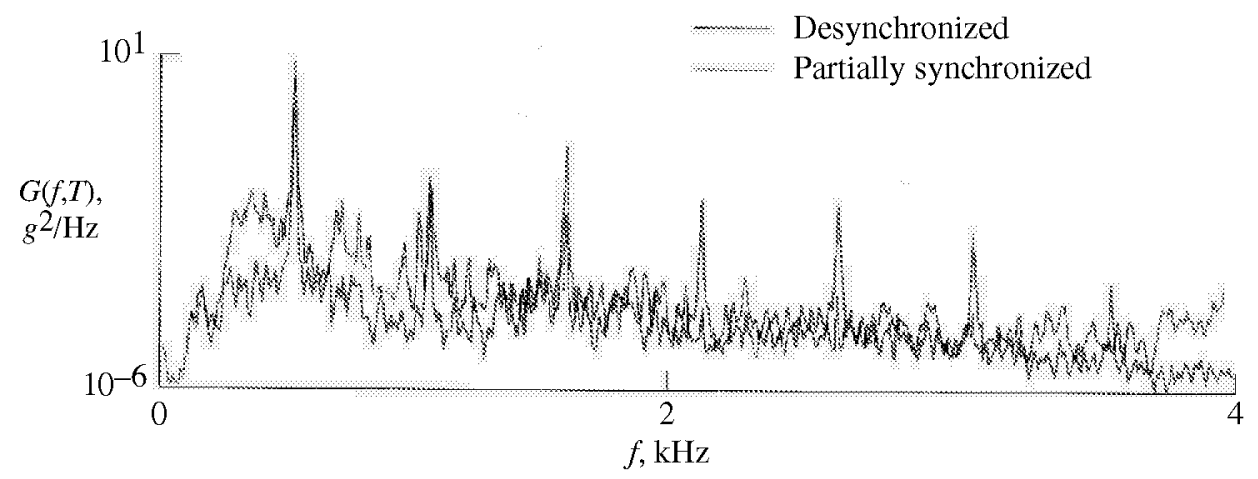

(c) Power spectral density with partially synchronized and desynchronized phases.

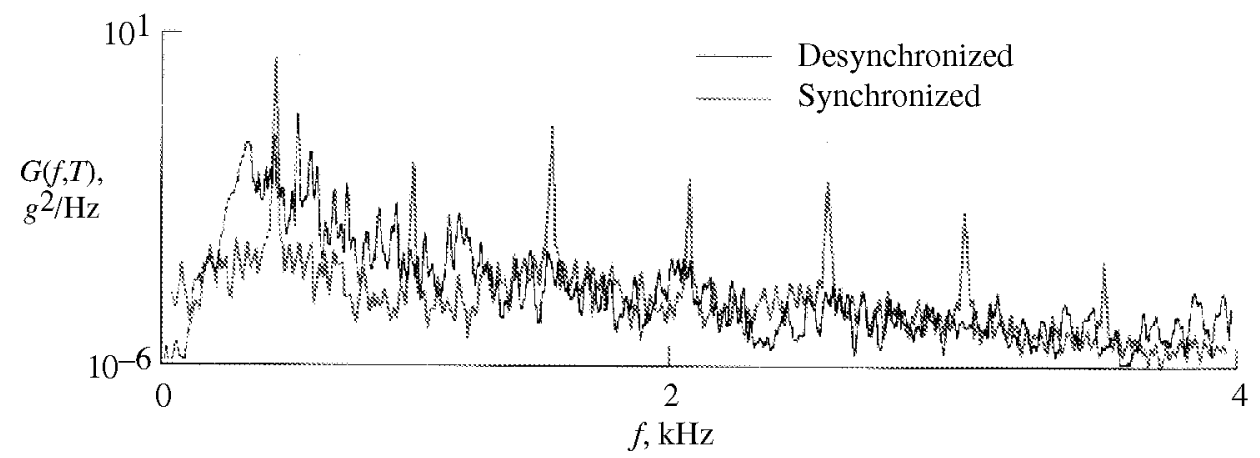

(d) Power spectral density with synchronized and desynchronized phases.

Figure 6. Response on panel A. 


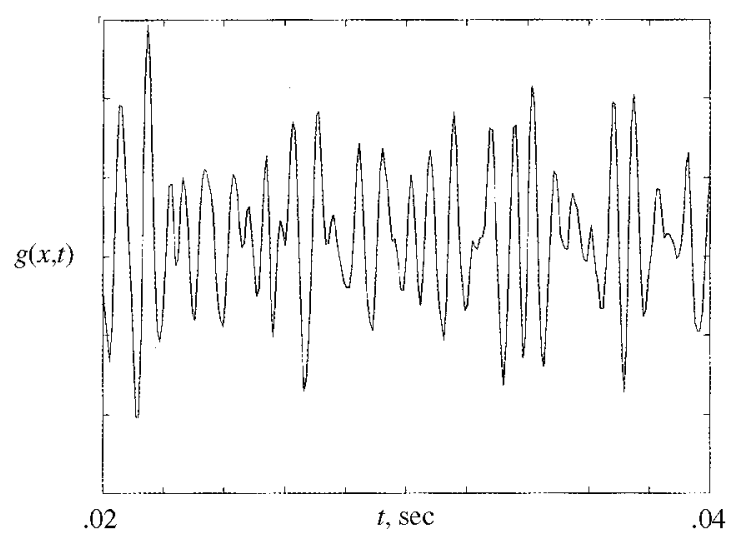

(a) Time series.

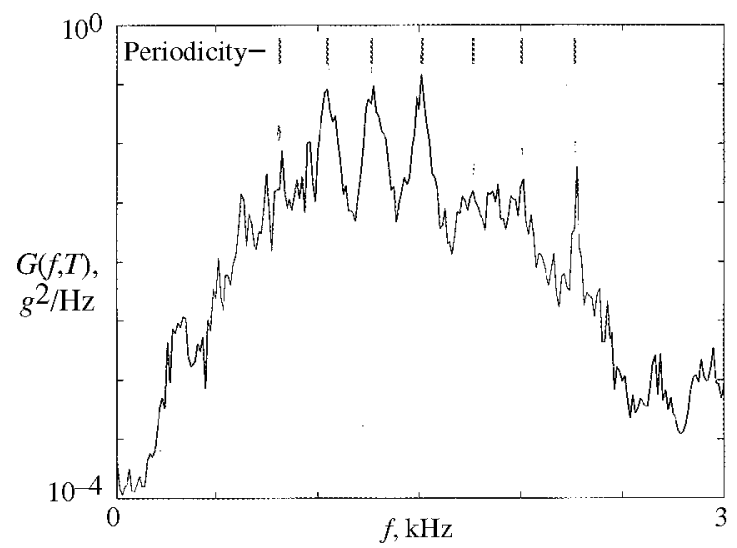

(b) Power spectra.

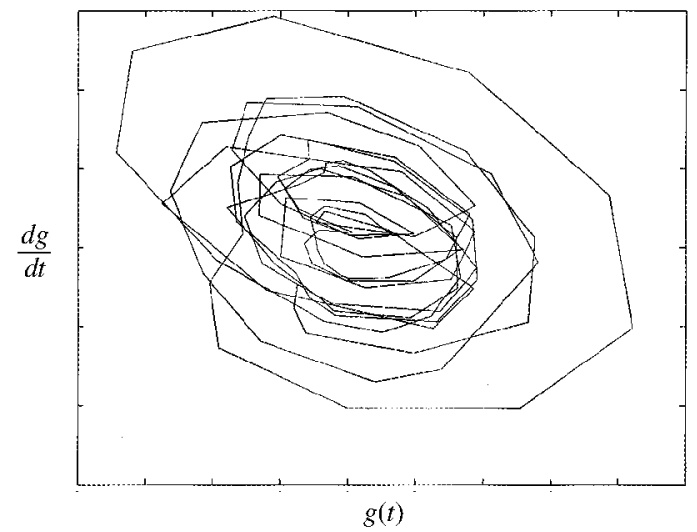

(c) Phase.

Figure 7. Panel response on Boeing MD-90 airplane at Mach number 0.80 and altitude of 10,000 m. 


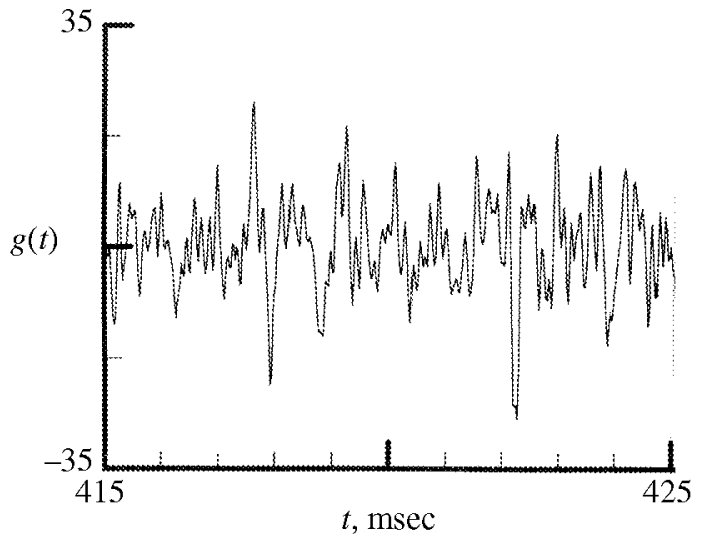

(a) Time series.

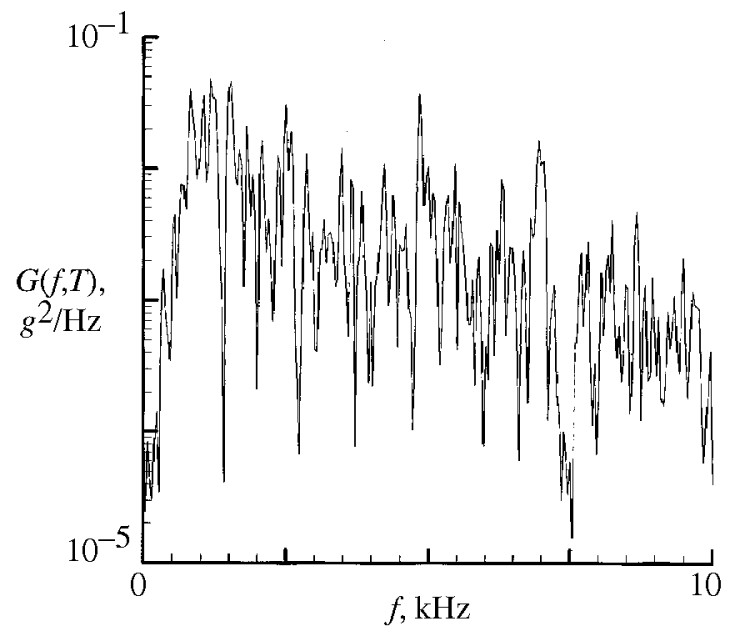

(b) Power spectra.

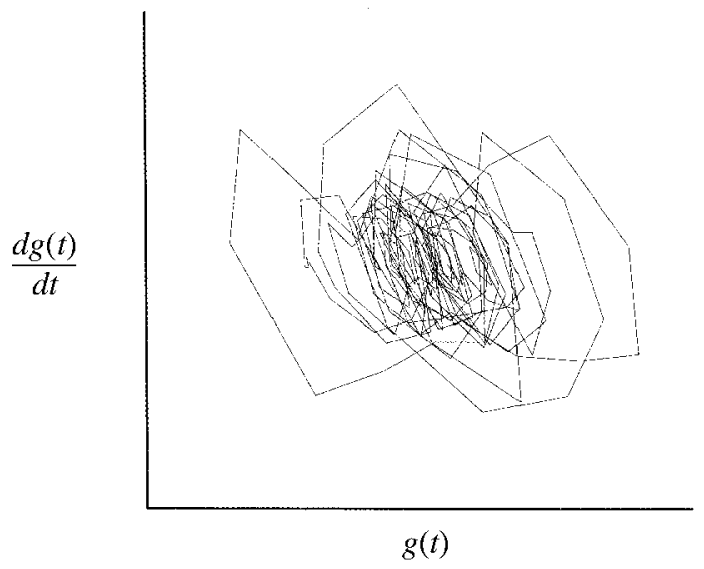

(c) Phase.

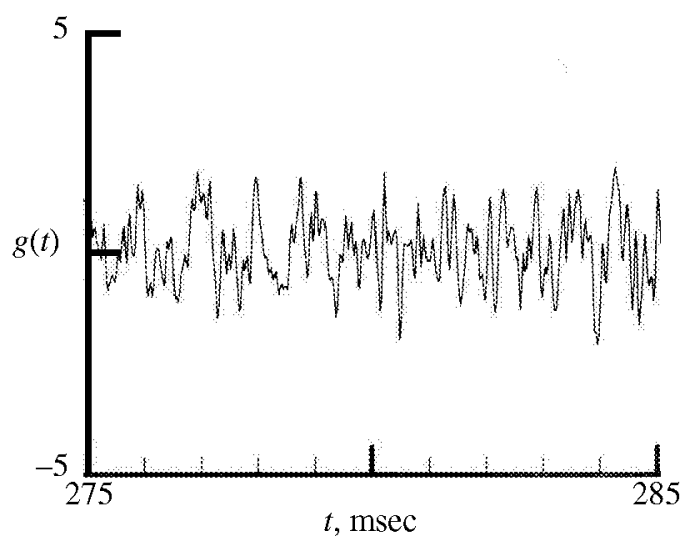

(d) Time series at $t=0.275-0.285$.

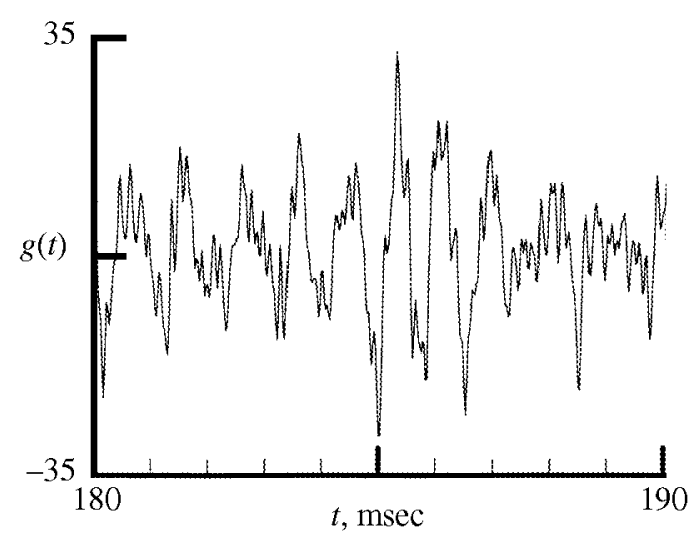

(e) Time series at $t=0.18-0.19$.

Figure 8. Panel response on Tu-144LL supersonic transport at Mach number 1.95 and altitude of 17,000 $\mathrm{m}$. 


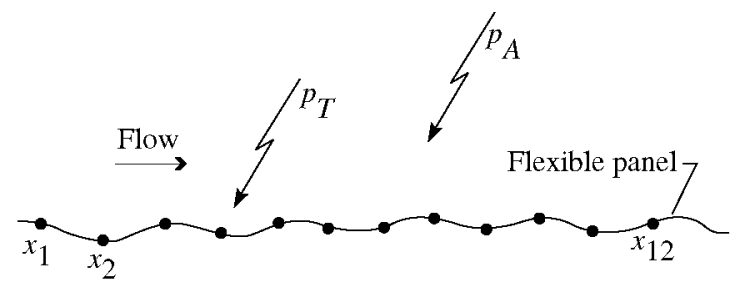

Figure 9. Analytical model.

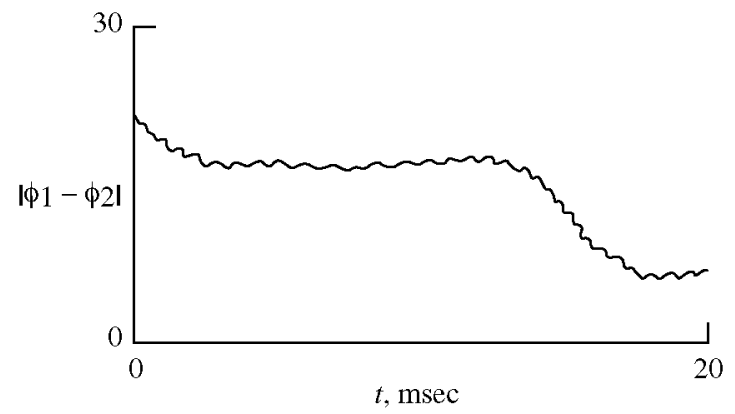

Figure 10. Phase difference of synchronous state versus time. 